\title{
Nutrition and physical activity in the prevention and treatment of sarcopenia: systematic review
}

\author{
C. Beaudart ${ }^{1,2} \cdot$ A. Dawson ${ }^{1}$ - S. C. Shaw ${ }^{1}$ - N. C. Harvey ${ }^{1}$. J. A. Kanis ${ }^{3,4} \cdot$ N. Binkley ${ }^{5}$.

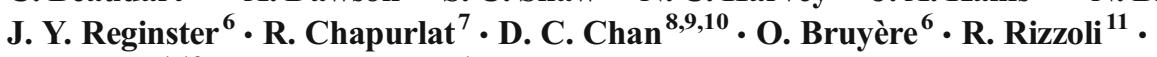 \\ C. Cooper ${ }^{1,12}$ - E. M. Dennison ${ }^{1}$ t the IOF-ESCEO Sarcopenia Working Group
}

Received: 9 January 2017 / Accepted: 31 January 2017 /Published online: 1 March 2017

(C) The Author(s) 2017. This article is published with open access at Springerlink.com

\begin{abstract}
Summary This systematic review summarizes the effect of combined exercise and nutrition intervention on muscle mass and muscle function. A total of 37 RCTs were identified. Results indicate that physical exercise has a positive impact on muscle mass and muscle function in subjects aged 65 years and older. However, any interactive effect of dietary supplementation appears to be limited.

Introduction In 2013, Denison et al. conducted a systematic review including 17 randomized controlled trials (RCTs) to explore the effect of combined exercise and nutrition intervention to improve muscle mass, muscle strength, or physical performance in older people. They concluded that further studies were needed to pro-
\end{abstract}

vide evidence upon which public health and clinical recommendations could be based. The purpose of the present work was to update the prior systematic review and include studies published up to October 2015.

Methods Using the electronic databases MEDLINE and EMBASE, we identified RCTs which assessed the combined effect of exercise training and nutritional supplementation on muscle strength, muscle mass, or physical performance in subjects aged 60 years and over. Study selection and data extraction were performed by two independent reviewers. Results The search strategy identified 21 additional RCTs giving a total of 37 RCTs. Studies were heterogeneous in terms of protocols for physical exercise and dietary supplementation (proteins, essential amino acids, creatine, $\beta$-hydroxy- $\beta$ -
This paper has been endorsed by the Committee of Scientific Advisors of the IOF.

\section{Cooper}

cc@mrc.soton.ac.uk

1 MRC Lifecourse Epidemiology Unit, University of Southampton, Southampton General Hospital, Southampton SO16 6YD, UK

2 Department of Public Health, Epidemiology and Health Economics, University of Liège, Liège, Belgium

3 Centre for Metabolic Bone Disease, Medical School, University of Sheffield, Sheffield, UK

4 Institute for Health and Aging, Catholic University of Australia, Melbourne, Australia

5 University of Wisconsin Osteoporosis Clinical Center and Research Program, Madison, WI, USA
6 Department of Public Health, Epidemiology and Health Economics, University of Liège, Liège, Belgium

7 INSERM UMR 1033, Université de Lyon, Hôpital E Herriot, Lyon, France

8 Department of Geriatrics and Gerontology, National Taiwan University Hospital, Taipei, Taiwan

9 Department of Internal Medicine, National Taiwan University Hospital, Taipei, Taiwan

10 Superintendent's Office, National Taiwan University Hospital Chu-Tong Branch, Hsinchu City, Taiwan

11 Service of Bone Diseases, Geneva University Hospitals and Faculty of Medicine, Geneva, Switzerland

12 NIHR Musculoskeletal Biomedical Research Unit, Institute of Musculoskeletal Sciences, University of Oxford, Oxford, UK 
methylbuthyrate, vitamin D, multi-nutrients, or other). In $79 \%$ of the studies (27/34 RCTs), muscle mass increased with exercise but an additional effect of nutrition was only found in 8 RCTs $(23.5 \%)$. Muscle strength increased in $82.8 \%$ of the studies (29/35 RCTs) following exercise intervention, and dietary supplementation showed additional benefits in only a small number of studies (8/35 RCTS, 22.8\%). Finally, the majority of studies showed an increase of physical performance following exercise intervention (26/28 RCTs, 92.8\%) but interaction with nutrition supplementation was only found in $14.3 \%$ of these studies (4/28 RCTs).

Conclusion Physical exercise has a positive impact on muscle mass and muscle function in healthy subjects aged 60 years and older. The biggest effect of exercise intervention, of any type, has been seen on physical performance (gait speed, chair rising test, balance, SPPB test, etc.). We observed huge variations in regard to the dietary supplementation protocols. Based on the included studies, mainly performed on wellnourished subjects, the interactive effect of dietary supplementation on muscle function appears limited.

Keywords Dietary · Intervention · Physical activity · Sarcopenia

\section{Introduction}

Sarcopenia has been defined by the European Working Group on Sarcopenia in Older People as a progressive and general loss of muscle mass and muscle function (defined either by a low muscle strength or a low physical performance) with advancing age [1]. Even though the loss of both is a natural part of the aging process, sarcopenia is defined when muscle mass and function falls below defined thresholds. Diagnosis of sarcopenia requires, therefore, the measurement of muscle mass, muscle strength, and physical performance [2]. Sarcopenia is recognized as a major public health problem $[3,4]$ due to significant clinical, economic, and social consequences. The implementation of preventive and therapeutic interventions has become a challenge due to the growing number of older persons affected by sarcopenia and its disabling complications.

Physical activity and nutritional supplementation have been investigated in several interventional studies. Recently, Cruz-Jentoft et al. [5] published a systematic review summarizing studies assessing the effect of physical activity and/or dietary supplementation on sarcopenia. Results indicated that most exercise trials showed an improvement of muscle strength and physical performance with physical activity, predominantly resistance training interventions. Results were consistent regarding the effect of dietary supplementation on muscle mass. Some studies have suggested a role of proteins, $\beta$-hydroxy $\beta$-methylbutyric acid, or amino acid on muscle function. However, the effects of these exercise and dietary interventions were assessed separately in this particular review; little is known about the combined effects of these two interventions. For this reason, Denison et al. [6] conducted a systematic review in 2013 to determine the effect of combined exercise and nutrition interventions on muscle mass, strength, and function in older people. That systematic review comprised 17 studies involving older ( $\geq 65$ years) adults published up to April 2013. The authors concluded that further studies were required to provide adequate evidence on which to base public health and clinical recommendations. The purpose of the present work was to provide an update to that systematic review by including studies published up to October 2015, and to focus on whether additional benefits arose if dietary supplementation was combined with exercise training.

\section{Methods}

\section{Literature search}

The literature search was performed in accordance with the Preferred Reporting Items for Systematic Reviews and Metaanalysis (PRISMA) statement. Using MEDLINE/Ovid and EMBASE/Ovid, we identified randomized controlled studies (RCTs) which assessed the combined effect of exercise training and nutritional supplementation on muscle strength, muscle mass, or physical performance. We updated the paper by Denison et al. [6] which limited the search strategy to February 2013. Therefore, we searched for any additional studies published between April 2013 and October 2015. The search strategy and search terms that were used for this research are detailed in Table 1. Additional studies were identified by a manual search of bibliographic references of relevant articles and existing reviews. Conference abstracts were not included.

\section{Study selection}

In the initial screening stage, two investigators independently reviewed the title and abstract for each of these references to exclude articles irrelevant to the systematic review. Rigorous inclusion criteria were adhered to (Table 2). In the second step, the two investigators independently read full texts of the articles not excluded in the initial stage, then selected the studies meeting the inclusion criteria (Table 2). All differences of opinion regarding selection of articles were resolved through discussion and consensus.

In order to maintain consistency between this update and the previous systematic review, the same inclusion criteria were used [6]. No age restriction was included in the search strategy but this review focused only on subjects aged 60 years and older. Studies performed on children, adolescents, and young adults were therefore excluded. Studies in which the nutritional intervention was energy restriction to promote 
Table 1 Search strategy (application to MEDLINE Ovid and EMBASE)

1. Sarcopenia/

2. Sarcopeni\$.tw

3. Muscle atrophy/

4. Muscle weakness/

5. Fat free mass.tw

6. Lean body mass.tw

7. Muscle mass.tw

8. Exp hand strength/

9. Grip strength.tw

10. Anthropometry/

11. Body composition/

12. Lean mass.tw

13. Or/ $1-12$

14. Exp exercise/

15. Exp Movement/

16. Muscle contraction/

17. Muscle Development/

18. Physical exertion/

19. Exp Physical endurance/

20. Exp muscle strength/

21. Physical fitness/

22. Exp Exercise test/

23. Exercise therapy.tw

24. Exp Exercise movement techniques/

25. Exp Psychomotor performance/

26. Muscle contraction/

27. Resistance exercise.tw

28. Aerobic exercice.tw

29. Endurance.tw

30. Physical exercise.tw

31. Physical performance.tw

32. Physical training.tw

33. Exercise programme.tw

34. Exercise technique.tw

35. Muscle mass.tw

36. Or/14-35

37. Nutrition.tw

38. Exp nutrition therapy/

39. Exp Nutritional physiological phenomena/

40. Exp Diet/

41. Exp Diet therapy/

42. Exp Dietary fats/

43. Exp Dietary proteins/

44. Exp Food/

45. Exp Food, fortified/

46. Exp Micronutrients/

47. Exp Dietary supplements/

48. Energy intake/

49. Nutrition.tw

50. Nutrition trial.tw

51. Dietary lipids.tw

52. Or/37-51

53. Randomized controlled trials/

54. Randomised controlled trial.tw

55. Randomized controlled trial.tw

56. Controlled clinical trial/

57. Controlled study.tw

58. Random allocation/

59. Random\$.tw

60. Randomly allocated.tw

61. Double blind method/

62. Single blind method/

63. Clinical trials.tw

64. Clinical trial/

65. Trial\$.tw

66. Intervention studies/
Table 1 (continued)

67. Intervention study.tw

68. Interventional study.tw

69. Placebo.tw

70. Placebo\$.tw

71. Or/53-70

72. And $/ 36,52$

73. And $/ 13,71,72$

74. (73 and humans/) or (73 not (humans/ or animals/))

75. Limit 74 to English language

76. Limit 75 to yr. $=$ "2013-Current" (344 results on PubMed (308 after deleting duplicates)- 859 with Embase (819 after remove duplicates)) total 992 after remove duplicates between the 2 databases

weight loss were also excluded. Finally, studies were also excluded if they included populations with a specific health condition (e.g., cirrhosis, cancer, diabetes, chronic kidney disease, etc.).

\section{Data extraction}

Data were extracted according to a standardized form to include authors, journal name, year of publication, country, objective of the study, length of intervention, type of population, gender ratio, mean age, age range, detailed groups with sample size, adherence to the treatment, $\%$ of participants who completed the study, adverse events, protocol of exercise intervention, protocol of nutritional intervention, muscle mass outcomes, muscle strength outcomes, and physical performance outcomes.

\section{Methodology quality assessment}

The quality of each study was independently assessed by two authors using the Jadad Score [7] system. The Jadad score

Table 2 Inclusion criteria

\begin{tabular}{ll}
\hline $\begin{array}{l}\text { Design } \\
\text { Participants }\end{array}$ & Randomized controlled trials \\
Exposure & Human, men, and women aged 60 years and older \\
& $\begin{array}{c}\text { Studies which include at least two groups of } \\
\text { comparison: a control group with only exercise } \\
\text { intervention and a treated group with combined } \\
\text { exercise intervention and nutritional intervention. } \\
\text { Exercise intervention can be resistance exercise, } \\
\text { aerobic exercise, or other. Nutrition intervention } \\
\text { involves the provision of nutrients supplied with } \\
\text { either a supplement or food }\end{array}$ \\
Outcome & Outcomes on muscle mass, on muscle strength, \\
& or on physical performance \\
Language & Studies published between April 2013 and \\
Date & end of October 2015 \\
\hline
\end{tabular}


ranges from 0 to 5 points. Studies were considered to be of excellent quality if the score was 5 , good quality if the score was 3 or 4 , and poor quality if otherwise.

\section{Presentation of results}

The findings were evaluated in a descriptive manner based on the information provided by each of the included studies. Because of the huge heterogeneity observed in the protocols of exercise and dietary supplementation, no meta-analysis was undertaken.

\section{Results}

\section{Included studies}

A total of 993 references were identified through the database search. A manual search of the bibliography of 10 relevant systematic reviews and meta-analyses did not generate any further studies for inclusion. After reading all titles and abstracts, 36 RCTs were selected for full-text review, following which 21 were included in this systematic review update. These 21 studies, added to the previous 17 considered by Denison et al. [6], gave a total of 37 RCTs included in the current systematic review (Fig. 1).

Characteristics of the studies are shown in Table 3. Twelve studies were performed in Europe, 11 in the USA/Canada, 7 in Asia, 4 in South America, and 3 in Australia. The number of participants ranged from 17 [35] to 222 [26] and study duration ranged from 4 weeks [27] to 18 months [42, 43]. The majority of studies included both male and female participants but 10 studies were confined to women only and 5 included only men. The mean age of participants varied from $59.5 \pm 4.5$ years $[9,10]$ to $87.1 \pm 0.6$ years $[39,40]$. Twelve RCTs were graded on the Jadad Scale as having an excellent quality, 15 a good quality, and 10 a poor quality.

Twenty-two studies used a two-group comparison methodology: one group receiving exercise + nutrition and the other group receiving exercise only (with placebo or no intervention). Eleven other studies used a four-group comparison model with one control group with no intervention, one group with exercise only, one group with nutrition only and finally, one group with combined exercise and nutrition interventions. Three other studies chose to randomize their population into three groups comprising a control group with no intervention, a group with exercise only, and a group with exercise combined with nutrition. Finally, one study used a five-group comparison model that included two groups with exercise and nutrition interventions, but used a different nutritional supplement in each of these two groups. For this systematic review, we used only results from two groups, one receiving exercise +
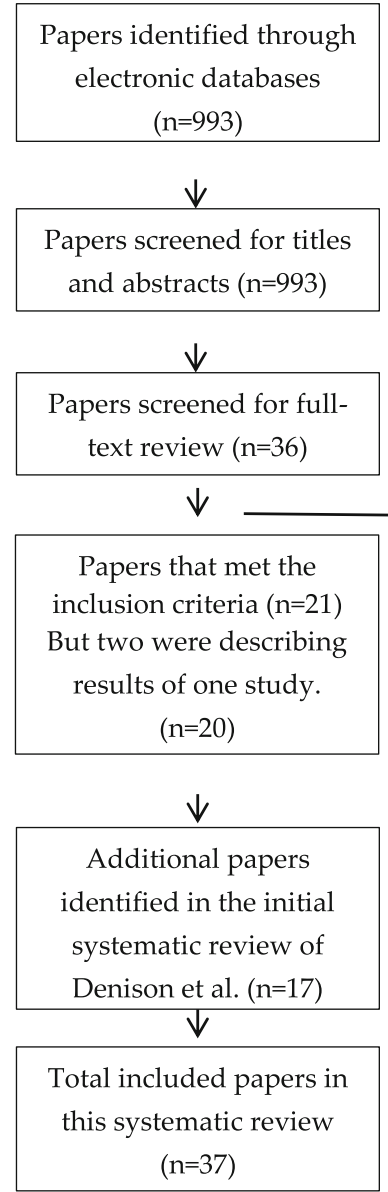

Fig. 1 Flow chart of literature search

nutrition and one receiving exercise only. It has to be noted that only half of the studies were double blinded.

Regarding nutritional interventions, 10 of the 37 studies used proteins. One further study used protein combined with essential amino acids, a second used protein combined with vitamin $\mathrm{D}$, and a third used protein combined with creatine. Three studies used essential amino acids alone, five studies used creatine alone, three studies used $\beta$-hydroxy- $\beta$ methylbutyrate alone, and two used vitamin $\mathrm{D}$ alone. Of the remaining 12 studies, five used multi-nutrient supplements and six used other products (vitamin and mineral-enhanced dairy and fruit products, green tea, magnesium oxide, milk fat globule membrane, soy isoflavones, and tea catechin). For exercise, the majority of studies used resistance training with the remainder using multicomponent training involving both resistance and additional exercises such as walking, fitness, aerobics, balance, etc.

\section{Types of nutritional intervention}

Results of the interventions are summarized in Table 4. 


\section{Protein supplementation}

Thirteen individual studies assessed the impact of a combined protein supplement and exercise intervention on the muscle function of elderly people. Most of these studies were of good quality but four were of poor quality $[12,24,26,29]$. In three of the 13 studies, protein was combined with creatine [12], essential amino acids [24], or vitamin D [26]. Supplementation protocols were heterogeneous in terms of studied population, duration of study, and supplementation dose, which varied from 7.4 to $45 \mathrm{~g}$ of protein per day. Twelve studies assessed the effect of the interventions on muscle mass and/or muscle strength but only nine reported results on physical performance.

Muscle mass: Muscle mass increased significantly with exercise in 11 of the 12 included RCTs. An interactive effect of protein supplementation and exercise was reported in only three of these studies: one looked at frail individuals [33], a second has been performed in elderly sarcopenic men [25], and the third enrolled female retirement village residents whose protein supplementation was lean red meat [22]. One other study [8] reported an increase of fat-free mass and appendicular lean mass only in the group supplemented with protein and exercise but the difference between the groups was not described. Muscle strength: All studies showed a significant improvement of leg muscle strength with exercise. No additional effect of protein was seen in the majority of these studies with the exception of three studies, each one of excellent quality: Daly et al. [22] showed significant improvement in leg extension in the group receiving lean red meat ( $45 \mathrm{~g}$ of protein/day) and exercise compared to an exercise-only group and Chalé et al. [17], who showed greater improvement in knee extensor peak power after a supplementation of $40 \mathrm{~g}$ of protein/day and, finally, Zdzieblik et al. [25] reported that quadriceps strength of the right leg (effect on the left leg was not assessed) increased more in the group taking $15 \mathrm{~g}$ of collagen peptide as supplement/day. Improvement in handgrip strength was seen in one study [33] but was absent in three others [20, 24, 29]. Finally, one study [8] reported an increase of the 1 repetition maximum (1RM) knee extensors only in the group with protein combined with exercise; however, the difference between the two groups was not reported. Physical performance: All studies showed a significant improvement of at least one physical performance test with exercise. No studies showed a significant difference between the groups receiving exercise only compared to the group receiving exercise combined with protein.

Summary: Muscle mass increased with exercises in 11/12 RCTs but an additional effect of protein was found in only 3/ 12 RCTs; Muscle strength increased with exercises in 12/12 $R C T$ s but an additional effect of protein was found in only 3/ 12 RCTs; Physical performance increased with exercise, for at least one outcome, in 9/9 RCTs with no additional effect of protein.

\section{Essential amino acids supplementation}

Three studies used essential amino acids (EAA) supplementation, $6 \mathrm{~g} /$ day for 3 months in sarcopenic communitydwelling older women [36], $10 \mathrm{~g}$ /day for 4 weeks in older adults recruited from nursing homes and adult day-care centers [27], and $12 \mathrm{~g} /$ day for 12 weeks in older men [35]. One study was of poor quality [35]. All three assessed the effect of intervention on muscle mass and muscle strength and two also measured the effect on physical performance [27, 36].

Muscle mass: Two studies reported an increase of muscle mass with exercise but did not report any difference between the group receiving EAA supplements and the group who did not $[35,36]$. The third study did not report any increase of muscle mass, neither for subjects receiving exercises only, nor in the groups of subjects receiving a combination of exercise and EAA supplements [27]. Muscle strength: Knee extension increased with exercise in two studies but no interaction was found with EAA supplementation. In the third study, no effect on isometric leg strength was observed [27]. Physical performance: Walking speed [36] and timed up and go [27] tests improved with exercise with no additional effect of EAA supplements. Standing balance and chair-stand test did not improve with treatment [27].

Summary: Muscle mass and muscle strength increased with exercise in 2/3 RCTS with no additional effect of EAA; Physical performance (walking speed and SPPB test only) increased with exercise in $2 / 2 R C T$ s with no additional effect of EAA.

\section{$\beta$-hydroxy- $\beta$-methylbutyrate (HMB) supplementation}

Three studies of good quality used Ca-HMB $[13,16,37]$ as a dietary supplement. In all studies, the treated group received $3 \mathrm{~g}$ of $\mathrm{Ca}-\mathrm{HMB}$ per day. The study duration varied: 24 weeks [13], 12 weeks [37], and 8 weeks [16]. Participants were healthy ambulatory older adults in the first two studies $[13,37]$ and healthy adults confined to complete bed rest for 10 days for the latter [16]. All studies assessed the effect on muscle mass and muscle strength but only two assessed the effect of treatment on physical performance $[13,16]$.

Muscle mass: Effects of Ca-HMB supplementation on muscle mass were not consistent across the three studies. Fat-free mass significantly increased with exercise in one study but no difference was evident between the group with combined exercise + Ca-HMB and the group with exercise only [13] Moreover, a significantly greater increase in fat-free mass was 


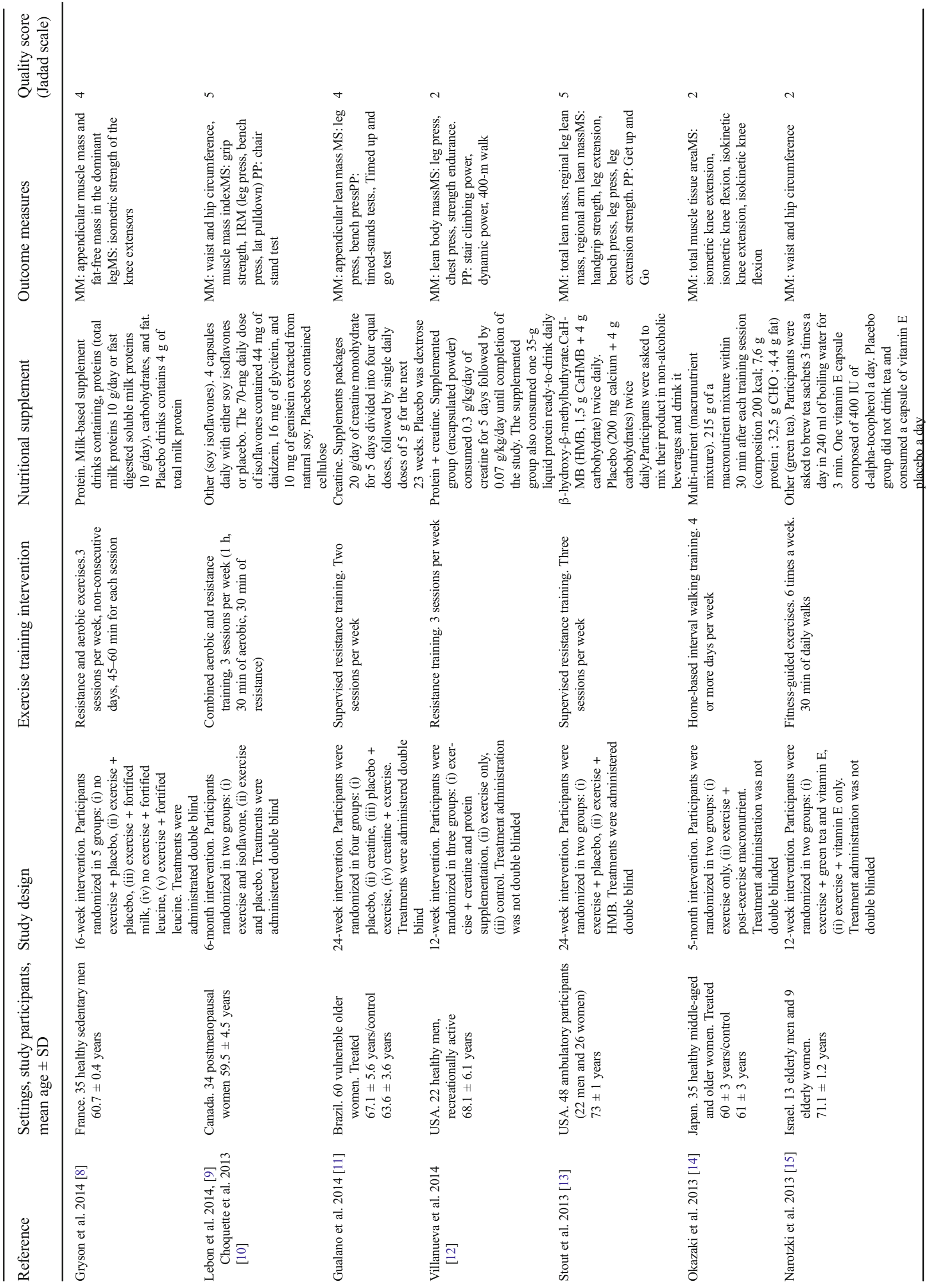




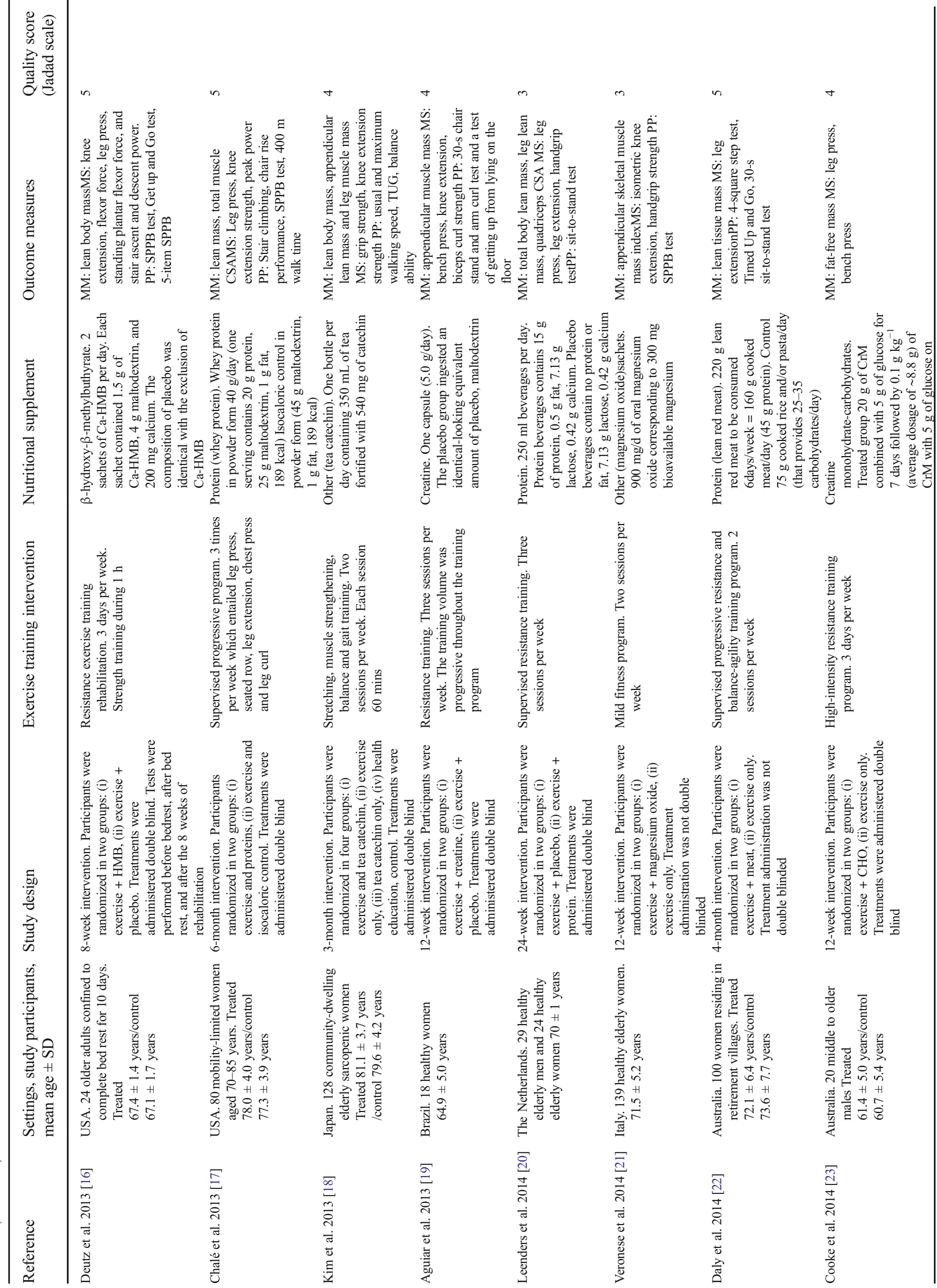




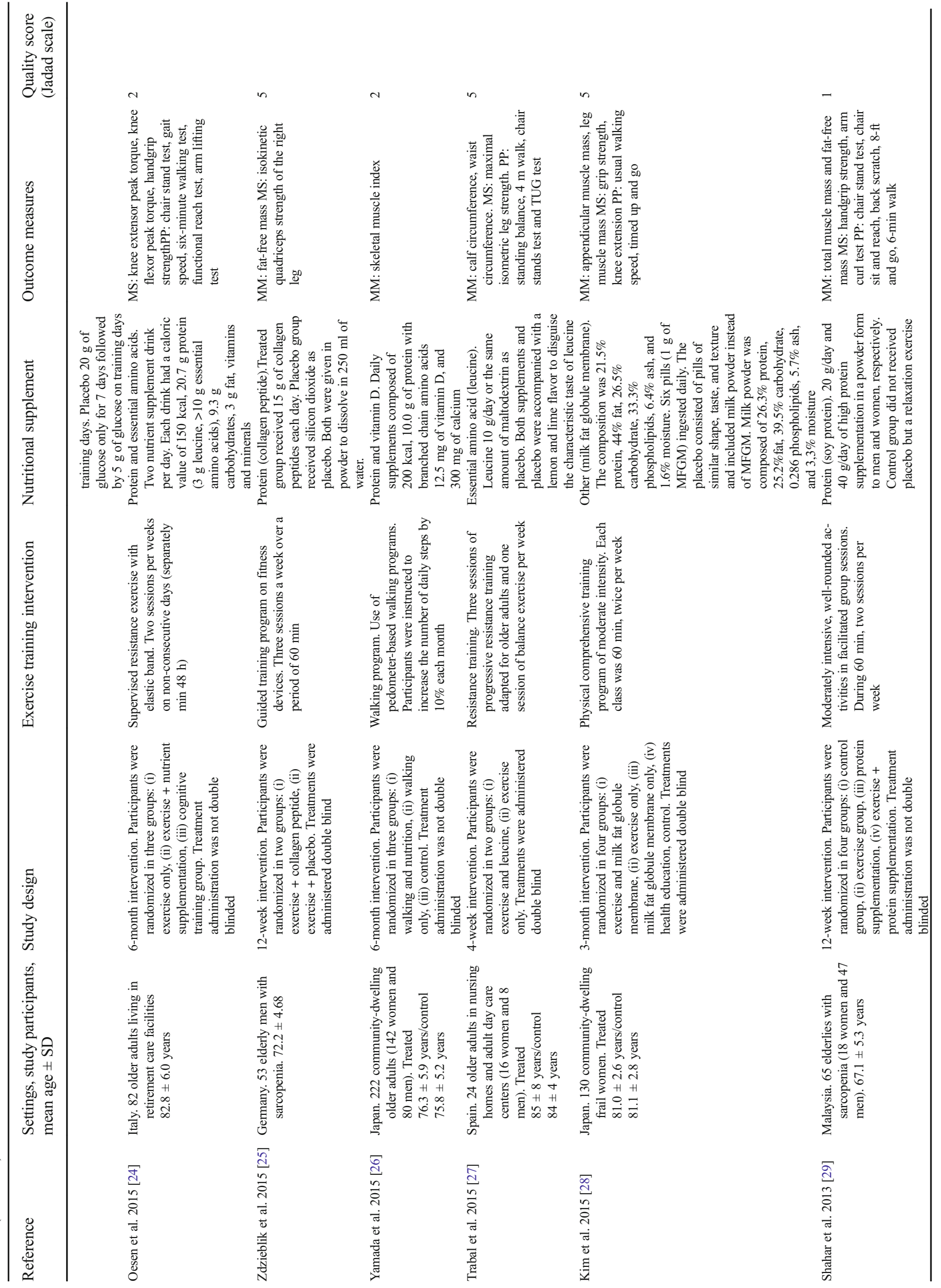




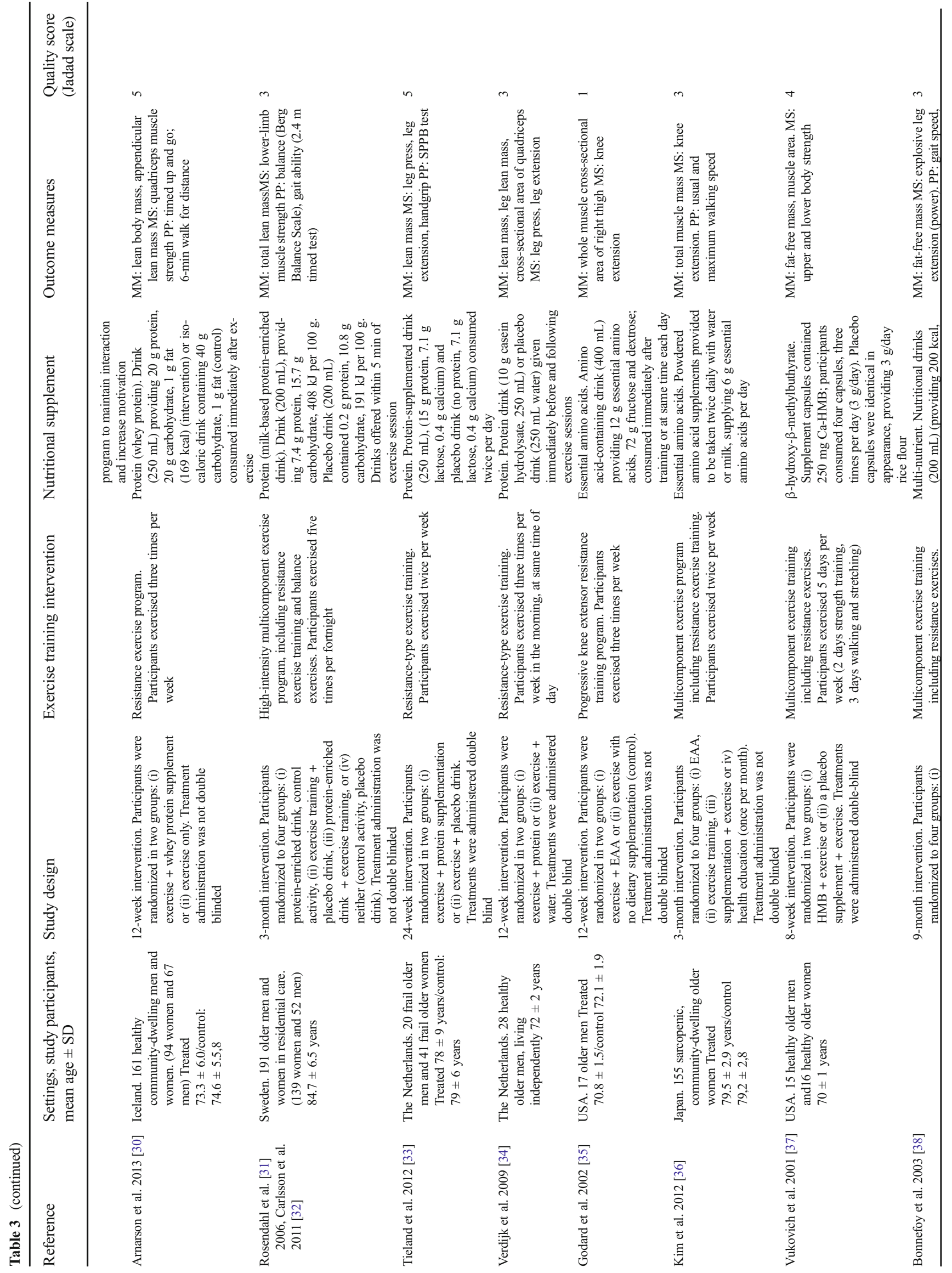




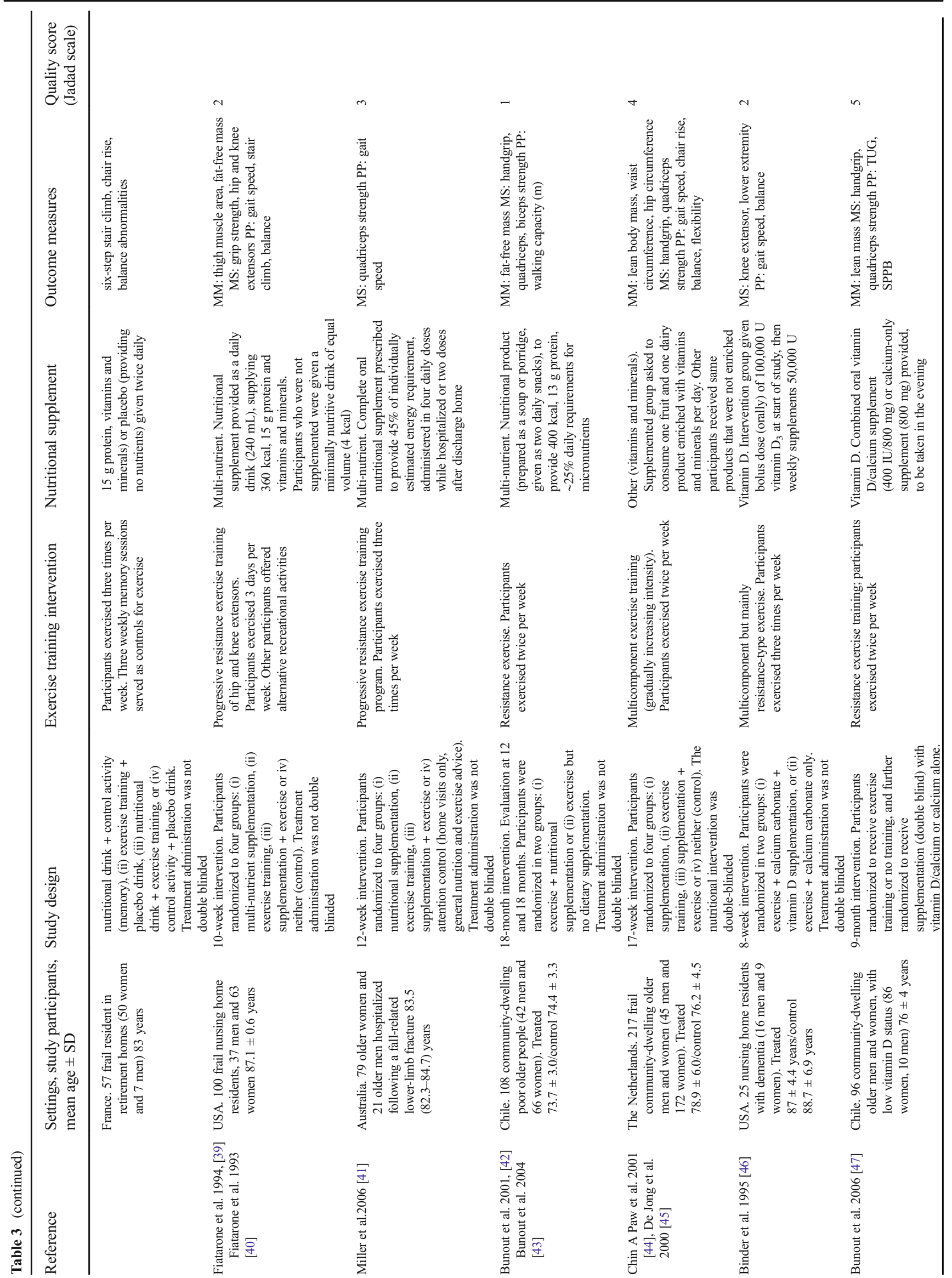


found in men from the placebo group. One study did not show any effect of the treatment on fat-free mass but did show an increase in thigh muscle area with exercise; no inter-group difference was seen [37]. Finally, the third study showed a significantly greater effect of exercise $+\mathrm{Ca}-\mathrm{HMB}$ in preventing the decline of lean body mass over a period of bed rest compared to exercise only [16]. Muscle strength: Muscle strength increased in two studies with exercise but no additional effect of $\mathrm{Ca}$ HMB was found. In the third study [37], no improvement in upper or lower body strength was found. Physical performance: The two studies showed an improvement in the performance of the Timed Up and Go test with exercise but did not show any added effect of nutritional supplementation and exercise. In a single study, no effect on the Short Physical Performance Battery (SPPB) test was found [16].

Summary: Muscle mass increased with exercise in $3 / 3$ $R C T s$ and an interactive effect of $H M B$ was found in $1 / 3$ RCTs; Muscle strength increased with exercises in 2/3 RCTs with no additional effect of HMB; Physical performance increased with exercise (TUG only) in $2 / 2$ RCTs with no additional effect of $H M B$.

\section{Multi-nutrient intervention}

Five studies reported results of treatment combining multinutrients and exercise on muscle strength; four of the five studies also looked at muscle mass and physical performance. Studies were performed on community-dwelling participants [14, 42, 43], frail retirement community residents [38], or nursing home residents $[39,40]$. The majority of studies were of poor quality [14, 39, 40, 42, 43].

Muscle mass: Two out of four studies did not report any improvement in fat-free mass with exercise or with exercise combined with multi-nutrient supplementation [38, 42, 43]. Two studies reported an increase in muscle mass with exercise $[14,39,40]$ but only in the cross-sectional area for the study of Fiatarone et al. [39, 40]. One of these failed to show any additional effect of nutritional supplementation [14] and the other did not describe the additional effect of multi-nutrient supplementation [39, 40]. Muscle strength: Results were heterogeneous for the five studies that assessed the combined effect of exercise and multi-nutrient supplementation on muscle strength. Two studies $[14,42]$ showed a significant improvement in muscle strength with exercise, one [42] did not report any additional effect of nutrition whereas the other study [14], reported greater improvement in isometric knee flexion in the group receiving combined exercise and multinutrient supplementation versus only exercise. Fiatarone et al. $[39,40]$ also reported an increase of muscle strength with exercise but did not describe the difference between the exercise only and combined multi-nutrient and exercise group. Finally, two other studies $[38,41]$ did not report any increase of muscle strength with treatment. Physical performance: 
Three studies described a significant improvement in physical performance with exercise. No additional effect of nutrition was seen in two studies [38, 42, 43]; one further study did not report whether there were any additional effect of nutrition $[39,40]$. Finally, a fifth study did not show any effect of treatment on gait speed [41].

Summary: Muscle mass increased with exercises in 2/4 RCTs with no additional effect of multi-nutrient; Muscle strength increased with exercises in $3 / 5 R C T s$ and an additional effect of multi-nutrient was found in 1/5 RCTs; Physical performance increased with exercise in 3/4 RCTs with no additional effect of multi-nutrient.

\section{Creatine supplementation}

Five good-quality studies have reported results of the effects of creatine supplementation on muscle mass and muscle strength; four of these also reported effects on physical performance. The protocols of supplementation were heterogeneous with three studies using $5 \mathrm{~g}$ /day of creatine while the two other studies used a higher dose of creatine for the first week followed by $5 \mathrm{~g} /$ day in one of the study and $0.1 \mathrm{~g} / \mathrm{kg} /$ day in the second study. The study duration varied from 12 weeks to 6 months.

Muscle mass: four out of the 5 studies showed greater improvement of muscle mass in the group treated with the combination of exercise and creatine compared to the control group with exercise only. The other study showed a significant increase in muscle mass with exercise but without any additional effect of creatine supplementation. Muscle strength: Muscle strength improved with exercise in all studies, with the exception of handgrip strength, which remained unchanged in one study [48]. Several studies found additional effects of creatine supplementation in addition to exercise on: bench press [11, 19, 23], knee extension [19, 48, 49], biceps curl performance [19], leg press [19], ankle dorsiflexion [48], isokinetic muscle strength [49], and, finally, endurance of knee extension [49]. Physical performance: Results were less consistent regarding physical performance. Two studies [48, 49] reported an improvement in physical performance with exercise but did not report any additional effects of creatine supplementation. One study reported no improvement in physical performance with either exercise or exercise plus creatine [11]. The final study found a greater improvement in the 30-s chair stand test and in an exercise where participants raised themselves from the floor, in the group receiving combined exercise and creatine [19].

Summary: Muscle mass increased with exercises in 5/5 $R C T s$ and an additional effect of creatine was found in $4 / 5$ RCTs; Muscle strength increased with exercises in 5/5 RCTs and an additive effect of creatine was found, for some of the muscle strength outcomes, in 4/5 RCTs; Physical performance increased with exercises in $3 / 4 R C T$ s and an interactive effect of creatine was found in $1 / 4$ RCTs.

\section{Vitamin D supplementation}

Two studies [46, 47] reported effects of combined exercise and vitamin $D_{3}$ supplementation on muscle strength and physical performance. One of those studies also reported effect on arm, waist and hip circumferences as well as lean mass [47]. The vitamin $\mathrm{D}_{3}$ dose was $400 \mathrm{IU} /$ day for 9 months in the study of Bunout et al. [47] and 50,000 IU/week (after an initial injection of 100,000 IU at study entry) for 8 weeks for the study of Binder et al. [46]. This last study was graded as having a poor quality [46] whereas the study of Bunout et al. [47] was a good-quality study.

Muscle mass: Only one study reported results on muscle mass [47]. No effects of exercise alone or of exercise combined with vitamin D supplementation were observed. Indeed, no significant changes in weight, circumferences, or body composition measured by DXA were observed in any of the groups. Muscle strength: Both studies reported significant improvement in muscle strength with exercise but did not report any difference between the exercise-only group and the group with combined exercise and vitamin D supplementation. Physical performance: Binder et al. [46] reported improved balance with exercise in a population of elderly nursing home residents with dementia. No additional effect of vitamin D supplementation was found and no improvement in gait speed was evident in either group. Bunout et al. [47] reported a significant improvement in the Timed Up and Go test for the group with combined vitamin D and exercise but no difference for the SPPB test between groups.

Summary: Muscle mass did not improve with exercise and no additional effect of vitamin D was found; Muscle strength increased with exercise in $2 / 2 R C T$ with no additional effect of vitamin D; Physical performance increased, for some of the physical performance outcomes, in $2 / 2$ RCTs with no additional effect of vitamin $D$, except for TUG in $1 / 2$ RCTs.

\section{Other supplementation}

Our systematic review identified six studies of good quality that used other types of nutritional supplements: green tea in elderly men and women [15], magnesium oxide in healthy elderly subjects [21], milk fat globule membrane in frail women [28], soy isoflavones in frail older women $[9,10]$, vitamin and mineralenhanced dairy and fruit products in frail community-dwelling older people $[44,45]$ and finally, tea catechin in sarcopenic women [18]. Four studies were 12 weeks in length [15, 18, $21,28]$, one was 6 -months in length $[9,10]$, and the last one was 17 weeks in length $[44,45]$.

Muscle mass: A significant effect of exercise alone on muscle mass was seen in various studies: in waist and hip circumference in men in the green tea [15] study, hip circumference in the soy isoflavones $[9,10]$ study participants, lean mass in the vitamin and mineral-enhanced dairy and fruit products 


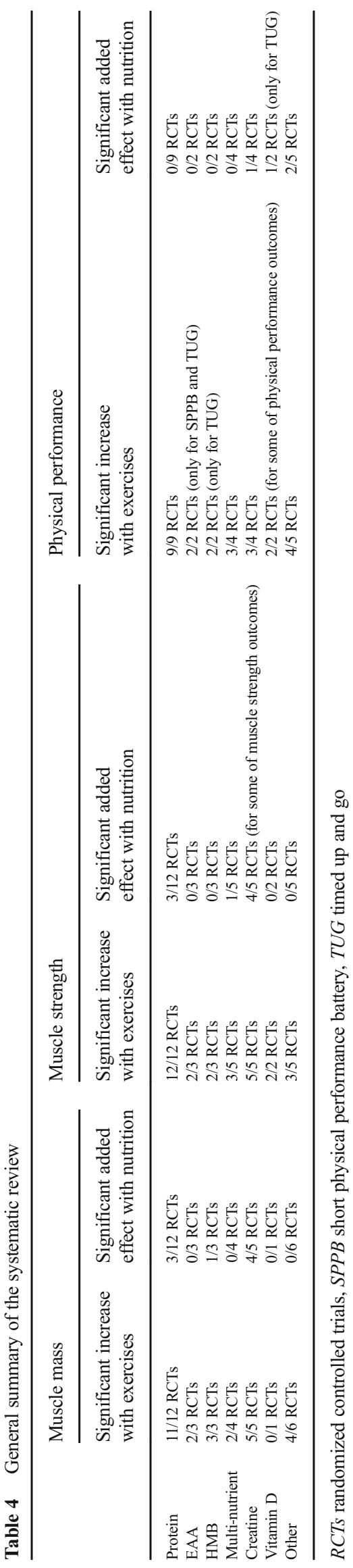

[44, 45]study, and leg lean mass in tea catechin [18]. No additional effects of nutritional supplements were observed across studies. Muscle strength: Exercise increased bench press and 1RM leg press without an additional effect of soy isoflavones $[9,10]$ and knee extension without additional effects of tea catechin [18]. A small increase of quadriceps strength was also shown in one study but the difference between groups was not described $[44,45]$. No effect of treatment was found on knee extension and handgrip strength in the other studies [9, 10, 21, 28]. Physical performance: Exercise combined with magnesium oxide significantly improved performance in the SPPB test, the chair stand test, and in the 4-m walking speed in the study of Veronese et al. [21]. TUG, usual gait speed, and maximum walking speed significantly improved in the exercise + tea catechin group compared to exercise group only in another study of Kim et al. [18] Walking speed and TUG test performance also improved with exercise in the study of Kim et al. [28] but no additional effect of milk fat globule membrane was described. In the study by Chin A Paw et al., improvements in physical performance were described but the additional effect of vitamin- and mineral-enhanced dairy and fruit products was not described [44, 45]. Finally, the chair stand test did not improve with treatment in two other studies $[9,10,18]$.

Summary: Muscle mass increased with exercise in 4/6 $R C T s$ and no additional effect of nutrition was found; Muscle strength increased with exercise in $3 / 5$ RCTs and no interactive effect of nutrition was found; Physical performance increased with exercise in $4 / 5 R C T$ s and an additional effect of nutrition was found in $2 / 5$ RCTs.

\section{Discussion}

This systematic review aimed to summarize results of RCTs assessing the effect of an intervention combining physical activity and dietary supplement on muscle mass and muscle function of subjects aged 60 years and older. Following a systematic review previously performed in 2013 by Denison et al. including 17 RCTs, we performed an update of this comprehensive systematic review and identified 21 RCTs published between April 2013 and October 2015. Thus, 37 RCTSs assessing the impact of a therapeutic intervention containing both physical activity and a nutritional supplement on muscle parameters were included in the present work. The study protocols were quite heterogeneous. Different types of physical activities have been studied in different populations that varied in sex, settings, and health status. Moreover, within each category of dietary supplements, the supplement dose and the length of study differed across RCTs.

Among the 37 RCTs included in the systematic review, 34 RCTs assessed the impact of intervention on muscle mass in elderly subjects. In almost $80 \%$ of the RCTs (27/ 
$34 \mathrm{RCTs}$ ), muscle mass increased with exercise training. In the majority of studies where no effect of exercise was observed, these were undertaken in frail subjects, residing in a nursing home, or, subjects with limited mobility. A hypothesis could be that the physical condition of these subjects did not allow them to perform the protocol for the physical activity intervention correctly. The majority of studies proposed three sessions per week. Fewer sessions may compromise efficacy of the physical activity intervention. An additional effect of nutritional intervention on muscle mass was only found in 8 RCTs (23.5\%), which were all of high quality ( 4 or 5 points in the Jadad Scale), 4 using creatine, 3 using proteins, and 1 using HMB as dietary supplement. The majority $(75 \%)$ of studies using creatine as a dietary supplement showed a higher effect on muscle mass once exercise intervention was combine with creatine. The combination of creatine supplementation and resistance training therefore seems to act synergistically. Only 3 of the 12 RCTs using protein as a dietary supplement reported an additional effect of protein when combined with physical activity. These three studies varied in type and dose of protein with no specific similarity between them that could explain their positive results as distinct from the other nine studies that did not report an effect on mass muscle with protein. Because of blunted response in muscle protein synthesis in older adults and reduced post-prandial inhibition of muscle protein breakdown, some authors recommended increasing protein intake to $1.2 \mathrm{~g} / \mathrm{kg}$ body weight/day in older adults and even more in frail older adults or elderly with acute or chronic disease $[50,51]$. Based on these recommendations, we hypothesized a beneficial effect of protein supplementation in muscle function in older people. However, it should be discussed that the baseline dietary intake of protein has not been reported in the different studies. Therefore, we do not know if the target of $1.2 \mathrm{~g} / \mathrm{kg} / \mathrm{day}$ has been reached or if differences could have been observed between populations who reached this target and those who did not. A meta-analysis published in 2012 [52] showed a positive effect of protein supplementation on muscle mass gains during prolonged resistancetype exercise training in older subjects. This probably means that the type of exercise training could have a non-negligible impact on results. Indeed, in this systematic review, inclusion criteria were not limited to one particular type of physical exercise. Finally, one out of the three studies using HMB as a dietary supplement also showed an intergroup difference between subjects undergoing exercise intervention and subjects undergoing a combination of exercise and dietary intervention. Of note, this study comprised subjects confined to bedrest. Subjects receiving a combination of exercise and HMB supplementation were more prevented for decline of lean body mass over bed rest compared to subjects undergoing exercises only. In this study, HMB supplementation did not increase muscle mass but prevented its decline.

Muscle strength increased in $82.8 \%$ of the studies (29/35 $\mathrm{RCTs}$ ) following an exercise intervention and, once again, dietary supplementation showed additional benefits in only a small number of studies (8/35 RCTS, $22.8 \%$ ) principally for creatine but only at specific muscle sites. In 4 out of the 5 RCTs using creatine as a dietary supplement (dose range 5$20 \mathrm{~g} /$ day), the group treated with the combination of exercise and dietary intervention showed greater improvement of muscle strength compared to exercise only. Three good-quality RCTs using protein as a dietary supplement also showed a greater effect on muscle strength when compared to the exercise group. These three studies were providing a high amount of protein with, respectively, 45, 40, and $15 \mathrm{~g}$ (collagen peptide) daily whereas the mean dose of supplementation in other studies was approximately $20 \mathrm{~g}$ /day. The dose of supplementation is likely to contribute to the inconsistent findings between studies. Handgrip strength, a component of sarcopenia definitions, was an outcome in 13 RCTs assessing the effect of a combined exercise and dietary intervention. Approximately half of the studies (6/13 RCTs) showed an improvement in grip strength with exercise. However, none of these RCTs showed an additional effect of dietary supplementation. Highlighted by these last results, it should be noted that, even if this systematic review revealed an increase of muscle strength following exercise in the majority of the studies, this seems particularly true for leg muscle strength.

A total of 29 RCTs also assessed the impact of combined physical activity and dietary supplementation on physical performance. We observed, in the majority of studies, an improvement in physical performance outcomes following an exercise intervention (26/28 RCTs, 92.8\%). In the two studies that did not report an improvement on physical performance, one was performed on frail people and the other one on hospitalized people. Physical performance was assessed using a variety of measures in the reported studies. The most commonly used measures were gait speed (used in 17 RCTs), followed by chair stand test (used in 13 RCTs), Timed Up and Go test (used in 8 RCTs), and SPPB test (used in 6 RCTs). The heterogeneity of both the type of exercise intervention and physical performance outcomes impedes general statements of findings on the association between exercise training and improvements in physical performance. Interaction of exercise and nutrition was found in only $17.8 \%$ of these studies (5/28 RCTs): one study when a multi-nutrient was used as a dietary supplement, another with creatine, a third study with vitamin D, another with tea catechin, and finally, one with magnesium oxide.

This study is an update of an existing systematic review and it followed the same rigorous methodology as the previous one. We searched multiple electronic databases to identify 
as many studies as possible that would meet our inclusion criteria. Nevertheless, this review is limited by the disparity between the studies. The exercise interventions described in the RCTs varied in regards of the types of exercises, doses, intensity, and duration. Moreover, adherence to these protocols were not reported, which impacts the assessment of the real effect of exercise on muscle features. Supplementations provided also varied, not merely for the dosage, but also for the duration, the way, and the frequency of administration. It must also be noted that half of these studies were not double blinded. These parameters are likely to be key factors in modulating the outcomes of studies investigating the potential benefit of dietary supplementation to further augment gains in muscle mass and strength during exercise training. Moreover, the majority of individual RCTs did not take into account the baseline nutritional status of the population. It makes perfect sense that elders, close to undernutrition in some of the dietary supplements presented, failed to respond properly to exercise training meant to increase their muscle mass and strength. Even a reasonable dose of the supplement may be insufficient if participants are very sick, frail, and/or malnourished at baseline. In the same vein, even if exercise could have a positive effect, nutritional supplement may not be effective in very healthy, fit, and/or vigorous elderly. Nutrition supplementation is likely to be more efficient if malnutrition is present. The specific elderly subpopulation should be regarded when evaluating the need for nutritional support during exercise training. Finally, even if our purpose was to assess combined effects of exercise training and dietary supplementation on muscle outcomes in sarcopenic subjects, a very limited number of included studies have been performed specifically on subjects affected by sarcopenia. Because of the condition of sarcopenic patients, it is likely that the effects observed in this systematic review would have been lesser in solely sarcopenic patients. It was however difficult for this study to focus only on sarcopenic subjects. Indeed, there are no universally accepted criteria for the diagnosis of sarcopenia in an operational sense. Therefore, we chose to focus on elderly subjects in a broader sense instead of focusing on one or more restricted definitions of sarcopenia.

In conclusion, physical exercise has a beneficial impact on muscle mass, muscle strength, or physical performance in healthy subjects aged 60 years and older. However, the additional effect of dietary supplementation has only been reported in a limited number of studies. For the majority of studies included in this systematic review, the population was composed of healthy older subjects. Studies assessing the impact of a combined exercise intervention and dietary intervention are still lacking in frail and sarcopenic populations, populations suffering from nutritional deficiency, or populations at risk of malnutrition. Further well-designed and wellconducted studies performed on these types of populations should be implemented. It seems likely that nutritional interventions in populations who are presenting nutritional or physical deficiencies would be more beneficial than interventions in well-nourished and replete populations. There is a need of a rigorous documentation of subject's baseline exercise level and nutritional status prior to implement intervention regimens in those future studies.

\section{Compliance with ethical standards}

Conflict of interest N Binkley received research support from Amgen, GE Healthcare, and Lilly, Merck and consultant/advisory board fees from Amgen, Astellas, Lilly, Merck, Nestle, and Radius. J-Y Reginster received consulting fees or paid advisory boards from Servier, Novartis, Negma, Lilly, Wyeth, Amgen, GlaxoSmithKline, Roche, Merckle, Nycomed-Takeda, NPS, IBSA-Genevrier, Theramex, UCB, Asahi Kasei, Endocyte, and Radius Health; lecture fees from Merck Sharp and Dohme, Lilly, Rottapharm, IBSA, Genevrier, Novartis, Servier, Roche, GlaxoSmithKline, Merckle, Teijin, Teva, Analis, Theramex, Nycomed, NovoNordisk, Ebewee Pharma, Zodiac, Danone, Will Pharma, Amgen, and PharmEvo; and grant support from Bristol Myers Squibb, Merck Sharp \& Dohme, Rottapharm, Teva, Roche, Amgen, Lilly, Novartis, GlaxoSmithKline, Servier, Pfizer, Theramex, Danone, Organon, Therabel, Boehringer, Chiltern, and Galapagos. ML Brandi is a consultant and grant recipient from Alexion, Abiogen, Amgen, Bruno Farmaceutici, Eli Lilly, MSD, NPS, Shire, SPA, and Servier. C Beaudart, A Dawson, S Shaw, N Harvey, JA Kanis, R Chapurlat, D Chan, O Bruyère, R Rizzoli, C Cooper, EM Dennison, G Adib, T Chevalley, P Clark, B Dawson-Hughes, A El Maghraoui, K Engelke, R Fielding, J Foldes, G Guglielmi, JM Kaufman, B Larijani, W Lems, L van Loon, G Lyritis, S Maggi, L Masi, E McCloskey, OD Messina, A Papaioannou, $\mathrm{P}$ Szulc, and N Veronese have nothing to declare.

\section{Appendix}

\section{the IOF-ESCEO Sarcopenia Working Group}

G. Adib

M. L. Brandi

T. Chevalley

P. Clark

B. Dawson-Hughes

A. El Maghraoui

K. Engelke

R. Fielding

A. J. Foldes

G. Gugliemi

J. M. Kaufman

B. Larijani

W. Lems

L. J. C. van Loon

G. P. Lyritis

S. Maggi

L. Masi

E. McCloskey

O. D. Messina

A. Papaioannou

P. Szulc

N. Veronese 
Open Access This article is distributed under the terms of the Creative Commons Attribution-NonCommercial 4.0 International License (http:// creativecommons.org/licenses/by-nc/4.0/), which permits any noncommercial use, distribution, and reproduction in any medium, provided you give appropriate credit to the original author(s) and the source, provide a link to the Creative Commons license, and indicate if changes were made.

\section{References}

1. Cruz-Jentoft AJ, Baeyens JP, Bauer JM et al (2010) Sarcopenia: European consensus on definition and diagnosis: report of the European Working Group on Sarcopenia in Older People. Age Ageing 39:412-423. doi:10.1093/ageing/afq034

2. Cooper C, Fielding R, Visser M et al (2013) Tools in the assessment of sarcopenia. Calcif Tissue Int 93:201-210. doi:10.1007/s00223-0139757-z

3. Beaudart C, Rizzoli R, Bruyère $O$ et al (2014) Sarcopenia: burden and challenges for public health. Arch Public Heal 72:45. doi:10. 1186/2049-3258-72-45

4. Bruyère $O$, Beaudart $C$, Locquet $M$ et al (2016) Sarcopenia as a public health problem. Eur Geriatr Med 7:272-275. doi:10.1016/j. eurger.2015.12.002

5. Cruz-Jentoft AJ, Landi F, Schneider SM, et al. (2014) Prevalence of and interventions for sarcopenia in ageing adults: a systematic review. Report of the International Sarcopenia Initiative (EWGSOP and IWGS). Age Ageing. doi: 10.1093/ageing/afu115

6. Denison HJ, Cooper C, Sayer AA, Robinson SM (2015) Prevention and optimal management of sarcopenia: a review of combined exercise and nutrition interventions to improve muscle outcomes in older people. Clin Interv Aging 10:859-869. doi:10.2147/CIA.S55842

7. Jadad AR, Moore RA, Carroll D et al (1996) Assessing the quality of reports of randomized clinical trials: is blinding necessary? Control Clin Trials 17:1-12

8. Gryson C, Ratel S, Rance M et al (2014) Four-month course of soluble milk proteins interacts with exercise to improve muscle strength and delay fatigue in elderly participants. J Am Med Dir Assoc 15(958):e1-e9. doi:10.1016/j.jamda.2014.09.011

9. Lebon J, Riesco E, Tessier D, Dionne IJ (2014) Additive effects of isoflavones and exercise training on inflammatory cytokines and body composition in overweight and obese postmenopausal women: a randomized controlled trial. Menopause 21:869-875. doi:10. 1097/GME.0000000000000177

10. Choquette S, Dion T, Brochu M, Dionne IJ (2013) Soy isoflavones and exercise to improve physical capacity in postmenopausal women. Climacteric 16:70-77. doi:10.3109/13697137.2011.643515

11. Gualano B, Macedo AR, Alves CRR et al (2014) Creatine supplementation and resistance training in vulnerable older women: a randomized double-blind placebo-controlled clinical trial. Exp Gerontol 53:7-15. doi:10.1016/j.exger.2014.02.003

12. Villanueva MG, He J, Schroeder ET (2014) Periodized resistance training with and without supplementation improve body composition and performance in older men. Eur J Appl Physiol 114:891905. doi:10.1007/s00421-014-2821-1

13. Stout JR, Smith-Ryan AE, Fukuda DH et al (2013) Effect of calcium $\beta$-hydroxy- $\beta$-methylbutyrate $(\mathrm{CaHMB})$ with and without resistance training in men and women $65+$ yrs: a randomized, double-blind pilot trial. Exp Gerontol 48:1303-1310. doi:10.1016/j.exger.2013.08.007

14. Okazaki K, Yazawa D, Goto M et al (2013) Effects of macronutrient intake on thigh muscle mass during home-based walking training in middle-aged and older women. Scand J Med Sci Sports 23: e286-e292. doi:10.1111/sms.12076
15. Narotzki B, Reznick AZ, Navot-Mintzer D et al (2013) Green tea and vitamin $\mathrm{E}$ enhance exercise-induced benefits in body composition, glucose homeostasis, and antioxidant status in elderly men and women. J Am Coll Nutr 32:31-40. doi:10.1080/07315724.2013.767661

16. Deutz NEP, Pereira SL, Hays NP et al (2013) Effect of $\beta$-hydroxy- $\beta$ methylbutyrate (HMB) on lean body mass during 10 days of bed rest in older adults. Clin Nutr 32:704-712. doi:10.1016/j.clnu.2013.02.011

17. Chalé A, Cloutier GJ, Hau C et al (2013) Efficacy of whey protein supplementation on resistance exercise-induced changes in lean mass, muscle strength, and physical function in mobility-limited older adults. J Gerontol A Biol Sci Med Sci 68:682-690. doi:10. 1093/gerona/gls221

18. Kim H, Suzuki T, Saito K et al (2013) Effects of exercise and tea catechins on muscle mass, strength and walking ability in community-dwelling elderly Japanese sarcopenic women: a randomized controlled trial. Geriatr Gerontol Int 13:458-465. doi:10. 1111/j.1447-0594.2012.00923.x

19. Aguiar AF, Januário RSB, Junior RP et al (2013) Long-term creatine supplementation improves muscular performance during resistance training in older women. Eur J Appl Physiol 113:987-996. doi:10.1007/s00421-012-2514-6

20. Leenders M, Verdijk LB, Van der Hoeven L et al (2013) Protein supplementation during resistance-type exercise training in the elderly. Med Sci Sports Exerc 45:542-552. doi:10.1249/MSS. 0b013e318272fcdb

21. Veronese N, Berton L, Carraro S et al (2014) Effect of oral magnesium supplementation on physical performance in healthy elderly women involved in a weekly exercise program: a randomized controlled trial. Am J Clin Nutr 100:974-981. doi:10.3945/ajen.113. 080168

22. Daly RM, O'Connell SL, Mundell NL et al (2014) Protein-enriched diet, with the use of lean red meat, combined with progressive resistance training enhances lean tissue mass and muscle strength and reduces circulating IL- 6 concentrations in elderly women: a cluster randomized controlled trial. Am J Clin Nutr 99:899-910. doi:10.3945/ajen.113.064154

23. Cooke MB, Brabham B, Buford TW et al (2014) Creatine supplementation post-exercise does not enhance training-induced adaptations in middle to older aged males. Eur J Appl Physiol 114:13211332. doi:10.1007/s00421-014-2866-1

24. Oesen S, Halper B, Hofmann M et al (2015) Effects of elastic band resistance training and nutritional supplementation on physical performance of institutionalised elderly - a randomized controlled trial. Exp Gerontol 72:99-108. doi:10.1016/j.exger.2015.08.013

25. Zdzieblik D, Oesser S, Baumstark MW et al (2015) Collagen peptide supplementation in combination with resistance training improves body composition and increases muscle strength in elderly sarcopenic men: a randomised controlled trial. Br J Nutr 114:1-9. doi:10.1017/S0007114515002810

26. Yamada M, Nishiguchi S, Fukutani N et al (2015) Mail-based intervention for sarcopenia prevention increased anabolic hormone and skeletal muscle mass in community-dwelling Japanese older adults: the INE (Intervention by Nutrition and Exercise) Study. J Am Med Dir Assoc 16:654-660. doi:10.1016/j.jamda.2015.02.017

27. Trabal J, Forga M, Leyes P et al (2015) Effects of free leucine supplementation and resistance training on muscle strength and functional status in older adults: a randomized controlled trial. Clin Interv Aging 10:713-723. doi:10.2147/CIA.S75271

28. Kim H, Suzuki T, Kim M et al (2015) Effects of exercise and milk fat globule membrane (MFGM) supplementation on body composition, physical function, and hematological parameters in community-dwelling frail Japanese women: a randomized double blind, placebo-controlled, follow-up trial. PLoS One 10:e116256. doi:10.1371/journal.pone.0116256

29. Shahar S, Kamaruddin NS, Badrasawi M et al (2013) Effectiveness of exercise and protein supplementation intervention on body 
composition, functional fitness, and oxidative stress among elderly Malays with sarcopenia. Clin Interv Aging 8:1365-1375. doi:10. 2147/CIA.S46826

30. Arnarson A, Gudny Geirsdottir O, Ramel A et al (2013) Effects of whey proteins and carbohydrates on the efficacy of resistance training in elderly people: double blind, randomised controlled trial. Eur J Clin Nutr 67:821-826. doi:10.1038/ejen.2013.40

31. Rosendahl E, Lindelöf N, Littbrand H et al (2006) High-intensity functional exercise program and protein-enriched energy supplement for older persons dependent in activities of daily living: a randomised controlled trial. Aust J Physiother 52:105-113

32. Carlsson M, Littbrand H, Gustafson Y et al (2011) Effects of highintensity exercise and protein supplement on muscle mass in ADL dependent older people with and without malnutrition: a randomized controlled trial. J Nutr Health Aging 15:554-560

33. Tieland M, Dirks ML, van der Zwaluw N et al (2012) Protein supplementation increases muscle mass gain during prolonged resistance-type exercise training in frail elderly people: a randomized, double-blind, placebo-controlled trial. J Am Med Dir Assoc 13:713-719. doi:10.1016/j.jamda.2012.05.020

34. Verdijk LB, Jonkers RAM, Gleeson BG et al (2009) Protein supplementation before and after exercise does not further augment skeletal muscle hypertrophy after resistance training in elderly men. Am J Clin Nutr 89:608-616. doi:10.3945/ajen.2008.26626

35. Godard MP, Williamson DL, Trappe SW (2002) Oral amino-acid provision does not affect muscle strength or size gains in older men. Med Sci Sports Exerc 34:1126-1131

36. Kim HK, Suzuki T, Saito K et al (2012) Effects of exercise and amino acid supplementation on body composition and physical function in community-dwelling elderly Japanese sarcopenic women: a randomized controlled trial. J Am Geriatr Soc 60:16-23. doi: 10.1111/j.1532-5415.2011.03776.x

37. Vukovich MD, Stubbs NB, Bohlken RM (2001) Body composition in 70-year-old adults responds to dietary beta-hydroxy-betamethylbutyrate similarly to that of young adults. J Nutr 131: 2049-2052

38. Bonnefoy M, Cornu C, Normand S et al (2003) The effects of exercise and protein-energy supplements on body composition and muscle function in frail elderly individuals: a long-term controlled randomised study. Br J Nutr 89:731-739. doi:10.1079/ BJN2003836

39. Fiatarone MA, O’Neill EF, Ryan ND et al (1994) Exercise training and nutritional supplementation for physical frailty in very elderly people. N Engl J Med 330:1769-1775. doi:10.1056/ NEJM199406233302501

40. Fiatarone MA, O'Neill EF, Doyle N et al (1993) The Boston FICSIT study: the effects of resistance training and nutritional supplementation on physical frailty in the oldest old. J Am Geriatr Soc 41:333-337

41. Miller MD, Crotty M, Whitehead C et al (2006) Nutritional supplementation and resistance training in nutritionally at risk older adults following lower limb fracture: a randomized controlled trial. Clin Rehabil 20:311-323

42. Bunout D, Barrera G, de la Maza P et al (2001) The impact of nutritional supplementation and resistance training on the health functioning of free-living Chilean elders: results of 18 months of follow-up. J Nutr 131:2441S-2446S

43. Bunout B, Barrera G, de la Maza P et al (2004) Effects of nutritional supplementation and resistance training on muscle strength in free living elders. Results of one year follow. J Nutr Health Aging 8:68-75

44. Chin A, Paw MJ, de Jong N, Schouten EG et al (2001) Physical exercise and/or enriched foods for functional improvement in frail, independently living elderly: a randomized controlled trial. Arch Phys Med Rehabil 82:811-817. doi:10.1053/apmr.2001.23278

45. de Jong N, Chin A, Paw MJ, de Groot LC et al (2000) Dietary supplements and physical exercise affecting bone and body composition in frail elderly persons. Am J Public Health 90:947-954

46. Binder, E.F. (1996) Implementing a structured exercice program for frail nursing home residents with dementia: issues and challenge. JAPA, vol 3(issue 4).

47. Bunout D, Barrera G, Leiva L et al (2006) Effects of vitamin D supplementation and exercise training on physical performance in Chilean vitamin D deficient elderly subjects. Exp Gerontol 41:746752. doi:10.1016/j.exger.2006.05.001

48. Brose A, Parise G, Tarnopolsky MA (2003) Creatine supplementation enhances isometric strength and body composition improvements following strength exercise training in older adults. $J$ Gerontol A Biol Sci Med Sci 58:11-19

49. Tarnopolsky M, Zimmer A, Paikin J et al (2007) Creatine monohydrate and conjugated linoleic acid improve strength and body composition following resistance exercise in older adults. PLoS One 2:e991. doi:10.1371/journal.pone.0000991

50. Nowson C, O'Connell S (2015) Protein requirements and recommendations for older people: a review. Nutrients 7:6874-6899. doi: 10.3390/nu7085311

51. Bauer J, Biolo G, Cederholm T et al (2013) Evidence-based recommendations for optimal dietary protein intake in older people: a position paper from the PROT-AGE Study Group. J Am Med Dir Assoc 14:542-559. doi:10.1016/j.jamda.2013.05.021

52. Cermak NM, Res PT, de Groot LCPGM et al (2012) Protein supplementation augments the adaptive response of skeletal muscle to resistance-type exercise training: a meta-analysis. Am J Clin Nutr 96:1454-1464. doi:10.3945/ajen.112.037556 